\title{
miR-509-3p Suppresses Migration, Invasion, and Epithelial- Mesenchymal Transition in Melanoma Cells by Targeting Collagen Triple Helix Repeat Containing 1
}

\author{
Keyu Yuan ${ }^{1} \oplus$, Yi $\operatorname{Sun}^{2} \oplus, \mathrm{Yu}^{2}{ }^{2} \oplus$ \\ 'Department of Plastic Surgery, Zhuji People's Hospital, Shaoxing City, Zhejiang Province, China \\ ${ }^{2}$ Department of Plastic Surgery, Zhejiang Provincial People's Hospital, Hangzhou City, Zhejiang Province, China
}

Background: microRNAs (miRNAs) are ubiquitously dysregulated in numerous tumor cell types, including melanoma cells. The anti-tumor effect of miR-509-3p was widely evaluated in various cancers.

Aims: To determine the functional role of miR-509-3p in melanoma. Study Design: Cell culture study.

Methods: Expression of miR-509-3p in melanoma cell models were assessed by qRT-PCR. Cell migration and invasion were analyzed by wound healing and transwell assays, respectively. Expression levels of biomarkers of epithelial-mesenchymal transition were determined by Western blot. Luciferase vectors containing wildtype or mutant miR509-3p binding site were constructed, and then dual-luciferase reporter assay.

Results: Dysregulated miR-509-3p level was found in melanoma cells. Elevated miR-509-3p expression suppressed melanoma cell migration $(P<.001)$ and invasion $(P<.001)$ capacities. Epithelial-mesenchymal transition of melanoma cells was repressed by miR-509-3p, along with increased $\alpha$-catenin/E-cadherin $(P<.001)$ and decreased vimentin/ fibronectin $(P<.001)$. CTHRC1 (collagen triple helix repeat containing 1) contained a potential binding site for miR-509-3p, and miR509-3p decreased protein expression of CTHRC1 in melanoma cells $(P<.001)$. CTHRC1 promoted melanoma cell migration and invasion $(P<.001)$, as well as contributed to epithelial-mesenchymal transition. Increased CTHRC1 expression attenuated miR-509-3p-induced inhibition of melanoma cell migration $(P<.001)$, invasion, and epithelialmesenchymal transition.

Conclusion: miR-509-3p suppressed the biological function of melanoma cells through negatively regulating CTHRC1, shedding light on miR-509-3p as a potential candidate for melanoma therapeutics and treatments.

\section{INTRODUCTION}

Considered the most serious type of skin cancer, melanoma is mainly derived from melanocytes in the skin, mucous membranes, nerve centers, and other regions. ${ }^{1}$ Melanoma causes high mortality due to its strong invasiveness, easy metastasis, and easy tolerance to the treatment. ${ }^{2}$ The current strategies targeting melanoma mainly rely on early surgical removal, and there are no sustained effective treatments for the advanced stages of melanoma. ${ }^{3}$ Investigation of the molecular mechanism underlying the pathogenesis, as well as the development of novel therapeutic strategies, are urgent for the prevention of malignant metastasis of melanocytes.
microRNAs (miRNAs) inhibit mRNA translation and regulate gene expressions. ${ }^{4}$ Due to the ability to regulate genes involved in cell proliferation, apoptosis, or migration, miRNAs have attracted more attention in tumor research. ${ }^{5}$ The role of miRNAs in the development and biology of melanocytes has been investigated recently, ${ }^{6}$ illustrating that miRNAs are implicated in the pathogenesis of melanoma. ${ }^{6}$ miRNAs have been reported to function as an either oncogenic or oncosuppressive mediator on melanoma, suggesting miRNAs as potential therapeutic targets. $^{7}$ The anticancer effect of miR-509-3p has been widely evaluated on renal cell carcinoma ${ }^{8}$ and ovarian cancer. ${ }^{9}$ Recently,

Address for Correspondence: Yu Ji, Department of Plastic Surgery, Zhejiang Provincial People's Hospital, Hangzhou City, Zhejiang Province, China e-mail: yu_ji123@126.com

Received: September 14, 2020 Accepted: January 8, 2021 •DOI: 10.5152/balkanmedj.2021.20049

Available at www.balkanmedicaljournal.org

ORCID iDs of the authors: K.Y. 0000-0003-1079-5145; Y.S. 0000-0003-1114-3165; Y.J. 0000-0003-1442-8430.

Cite this article as:

Yuan K, Sun Y, Ji Y. miR-509-3p suppresses migration, invasion, and epithelial-mesenchymal transition in melanoma cells by targeting collagen triple helix repeat containing 1. Balkan Med J. 2021; 38(3):177-182.

Copyright@Author(s) - Available online at http://balkanmedicaljournal.org/ 
miR-509-3p was differentially expressed in A375 with resistance to vemurafenib ${ }^{10}$ and melanoma patients. ${ }^{11}$ However, the functional role of miR-509-3p in melanoma has not been thoroughly elucidated yet.

CTHRC1 retards collagen I synthesis and promotes cell migration of fibroblasts, facilitating vascular remodeling and tissue repair. ${ }^{12}$ Considering the tight link between tissue repair and carcinogenesis, CTHRC1 was regarded as an oncogene in tumors. ${ }^{13}$ CTHRC1 has been found to be overexpressed in melanoma cells, thus contributing to melanoma metastasis. ${ }^{14} \mathrm{We}$ explored whether miR-509-3p regulated melanoma progression through targeting CTHRC1.

\section{MATERIAL AND METHODS}

\section{Cell Culture}

A375, SK-MEL-28, WM115, and WM35 (Chinese Academy of Sciences, Shanghai, China) were cultured in RPMI-1640 medium containing 10\% fetal bovine serum (Lonza, Basel, Switzerland) in a $37^{\circ} \mathrm{C}$ humidified incubator.

\section{Plasmid Construction and Cell Transfection}

CTHRC1 ORF was inserted into pcDNA3.1 backbone (Invitrogen, Waltham, MA, USA) to generate pcDNA-CTHRC1. SK-MEL-28 and WM115 were transfected with pcDNA vectors, miR-509-3p mimics, and an inhibitor (GenePharma, Shanghai, China) via Lipofectamine ${ }^{\mathrm{TM}} 2000$ (Invitrogen) for $48 \mathrm{~h}$.

\section{Cell Migration}

Transfected SK-MEL-28 and WM115 cells with 80-90\% confluence were scratched, and a wound field in each well was generated. After $24 \mathrm{~h}$ of post-wound scratching, the mobilized cells were evaluated by a microscope (Olympus CY3, Tokyo, Japan).

\section{Cell Invasion}

Upper chambers (Corning, NY, USA) were seeded with transfected SK-MEL-28, and WM115 cells were supplemented with a serumfree medium. Medium containing serum was added into the lower chambers. After $48 \mathrm{~h}$ post-seeding, the cells in the lower chambers were fixed with $4 \%$ methanol and stained with crystal violet before counting using a microscope.

\section{Western Blot}

Protein samples were extracted from transfected SK-MEL-28, and WM115 cells were extracted using RIPA lysis buffer (BioTeke, Beijing, China). Proteins $(30 \mu \mathrm{g})$ were separated using SDS-PAGE and transferred onto a polyvinylidene difluoride membrane. The membrane was blocked in 5\% skim milk and incubated with antiCTHRC1 (1:1000; Proteintech Group, Chicago, IL, USA), anti- $\alpha$ catenin (1:2000), anti-E-cadherin (1:2000), anti-vimentin (1:2500), anti-fibronectin (1:2500), or anti- $\beta$-actin (1:3000) antibodies. The membrane was incubated with horseradish peroxidase-conjugated secondary antibodies (1:5000) by using Super Signal West Pico Chemiluminescent Substrate kit (Thermo Fisher; Waltham, MA, USA) as per the manufacturer's instruction.

\section{Luciferase Reporter}

Wildtype or mutant of CTHRC1 3' UTR sequences was cloned into pMIR-GLO ${ }^{\text {TM }}$ Luciferase vector (Promega, Madison, WI, USA). SK-MEL-28 and WM115 were co-transfected with CTHRC1-WT or CTHRC1-MUT vector with miR-509-3p mimic or NC mimic. After $48 \mathrm{~h}$ post-transfection, cells were processed using the dualluciferase reporter assay system (Promega).

\section{qRT-PCR}

RNAs were extracted from transfected SK-MEL-28 and WM115 via TRIzol reagent (Invitrogen) and reverse transcribed into cDNAs with M-MLV RT kit (Promega). The relative expression level of miR-509-3p was evaluated by TaqMan microRNA assays (Thermo Fisher). Primers were listed as follows: miR-509-3p (F; 5'-GGGATTCTGCTCGTGTTCCA-3' and R: 5'-GGTTCTTACGGGCATCCACA-3') and U6 (F: 5'-GCTCGCTTCGGCAGCACA-3' and R: 5'-GAGGTATTCGCACCAGAGGA-3').

\section{Statistical Analysis}

Data with at least 3 repeats were expressed as the mean \pm standard deviation, analyzed by Student's $t$-test or one-way analysis of variance with a Tukey's post hoc test. $P<.05$ was considered statistically significant difference.

\section{RESULTS}

miR-509-3p Suppressed Malignant Metastasis of Melanoma To elucidate the role of miR-509-3p in melanoma, A375, SK-MEL-28, WM115, and WM35 cells were processed using qRT-PCR analysis. WM35 cells showed the highest miR-509-3p expression, while WM115 cells showed the lowest expression (Figure 1A). miR-509-3p expression was higher in A375 than that in SK-MEL-28 (Figure 1A). Therefore, SK-MEL-28 $(P=.001)$ and WM115 $(P<.001)$ were then processed using gain-of functional assays by overexpressing miR-509-3p mimic (Figure 1B). The migratory (Figure 1C) capacity of SK-MEL-28 $(P=.032)$ and WM115 $(P<.001)$ was repressed by miR-509-3p transfection, and the invasive (Figure 1D) capacity of SK-MEL-28 $(P=.002)$ and WM115 $(P=.003)$ cells were also repressed by miR-509-3p overexpression, suggesting that miR-509-3p repressed malignant metastasis of melanoma.

\section{miR-509-3p Repressed Epithelial-Mesenchymal Transition in Melanoma}

To elucidate the role of miR-509-3p in epithelial-mesenchymal transition, Western blot analysis of biomarkers was performed in SK-MEL-28 and WM115 cell models. Epithelial markers, including $\alpha$-catenin $(P<.001$ and $P=.0001)$ and E-cadherin $(P<.001$ and $P<.001)$, were increased followed by miR-509-3p overexpression in SK-MEL-28 and WM115 cells (Figure 2). Whereas mesenchymal markers, such as vimentin $(P<.001$ and $P<.001)$ and fibronectin $(P<.001$ and $P<.001)$, were decreased following miR-509-3p treatment (Figure 2), suggesting that miR-509-3p suppressed epithelial-mesenchymal transition in melanoma cells. 
A

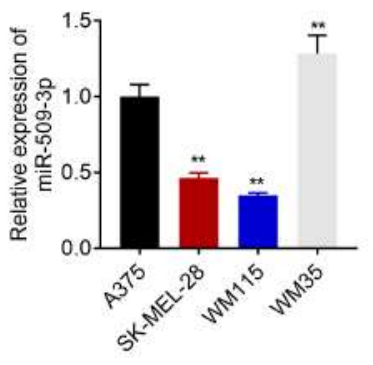

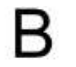

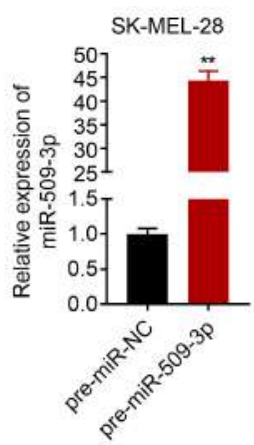

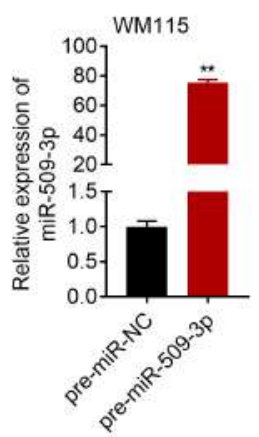

C

pre-miR-NC

pre-miR-509-3p

pre-miR-NC

pre-miR-509-3p

Oh
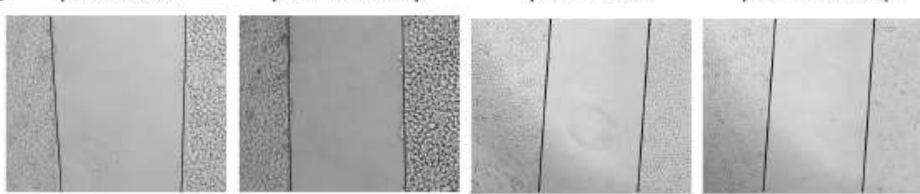

$24 h$
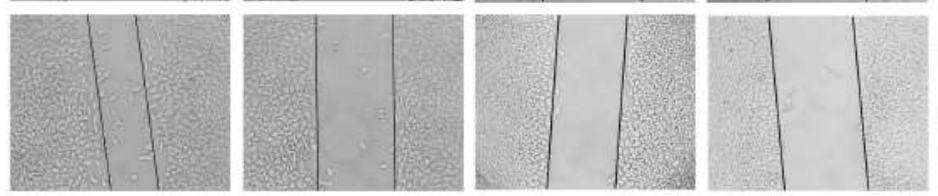

WM115

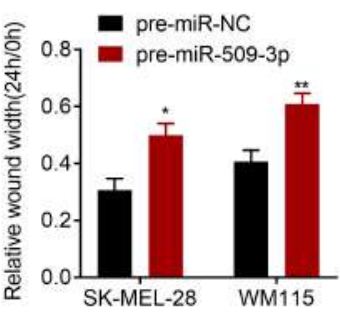

pre-miR-NC

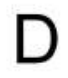

SK-MEL-28

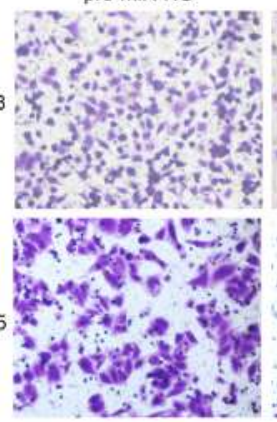

pre-miR-509-3p

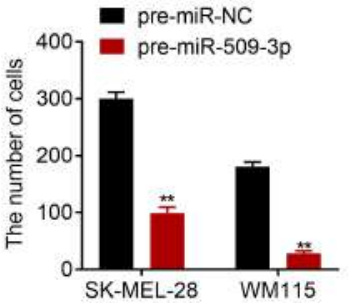

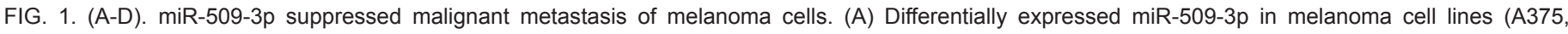

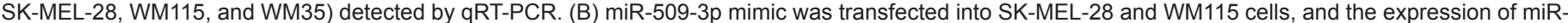

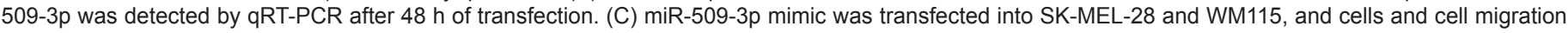

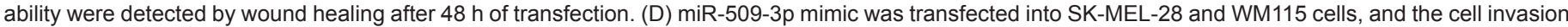
ability was detected by transwell after $48 \mathrm{~h}$ of transfection. ${ }^{* *} P<.01$.
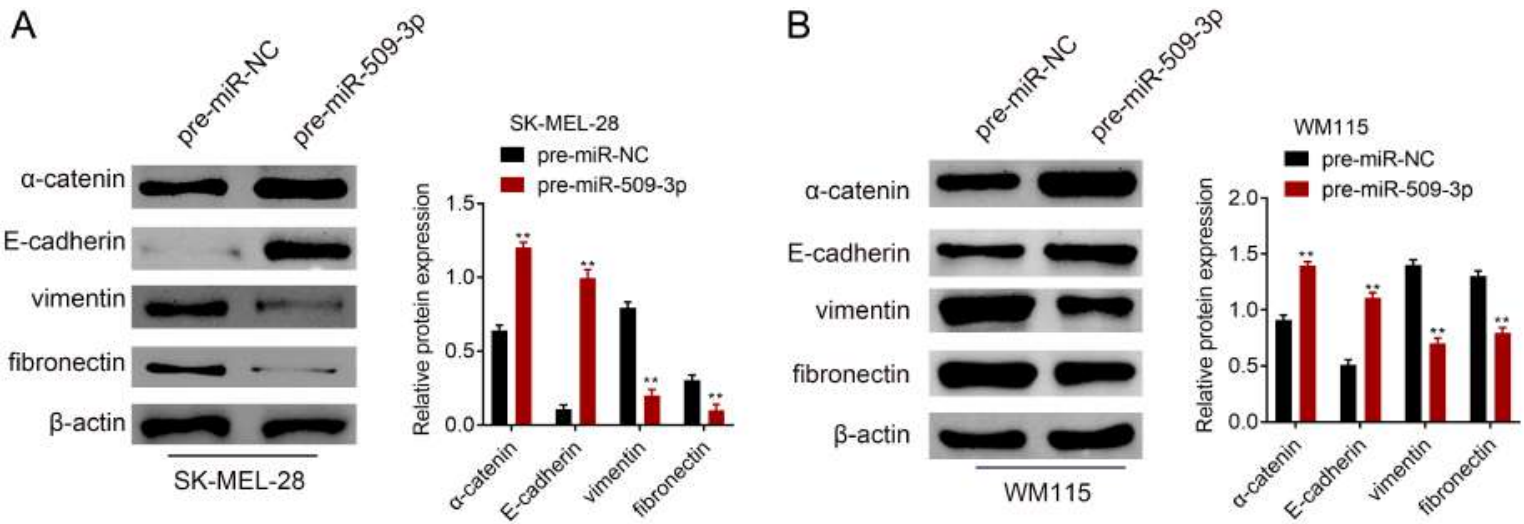

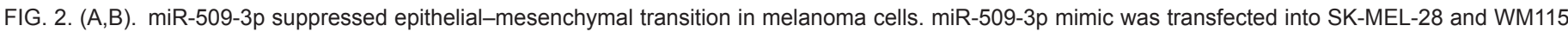
cells, and protein expression levels of $\alpha$-catenin, E-cadherin, vimentin, and fibronectin were detected by Western blot after $48 \mathrm{~h}$ of transfection. ${ }^{* \star} P<.01$. 

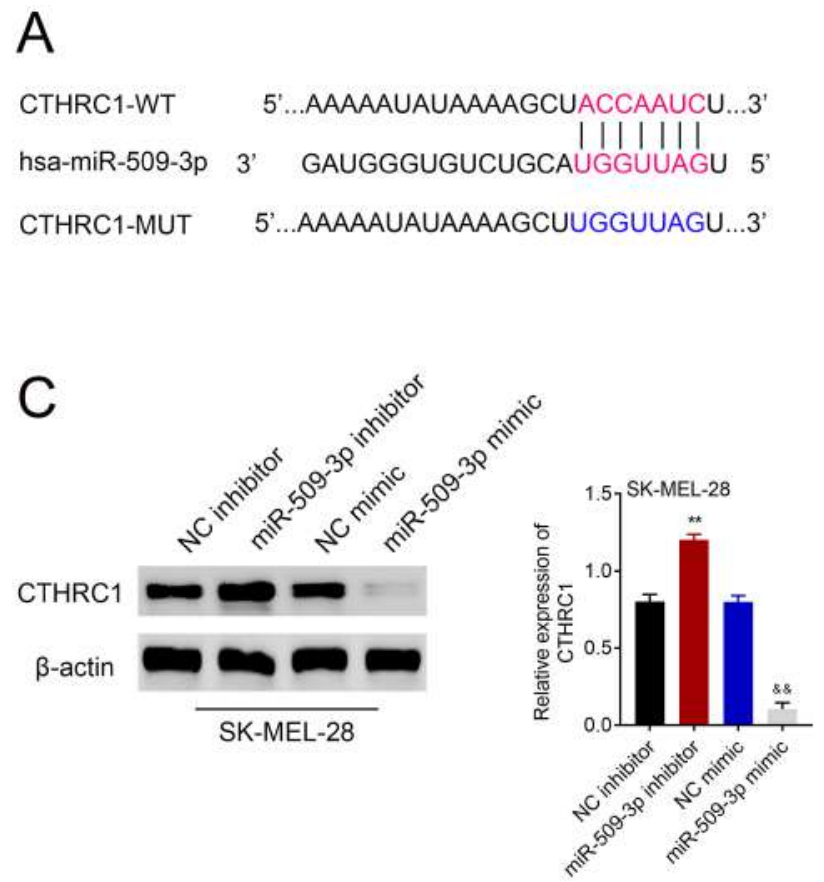

\section{$\mathrm{B}$}

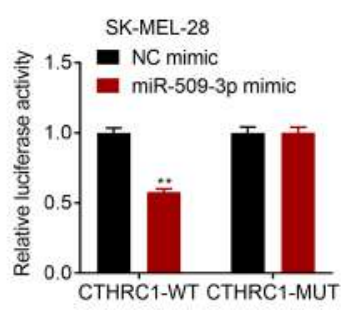

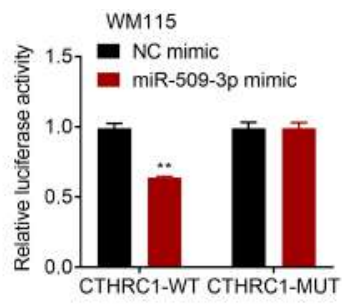

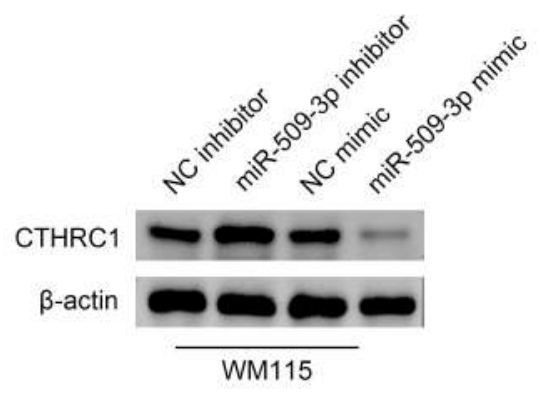

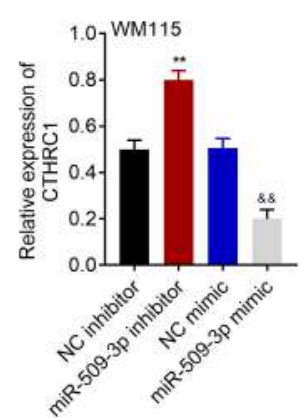

FIG. 3. (A-C). CTHRC1 was targeted by miR-509-3p in melanoma cells. (A) The potential binding site and mutant site between miR-509-3p and CTHRC1 by TargetScan. (B) Effect of miR-509-3p on luciferase activities of CTHRC1-WT and CTHRC1-MUT in SK-MEL-28 and WM115 cells were detected by luciferase activity assay. (C) miR-509-3p mimic or inhibitor was transfected into SK-MEL-28 and WM115 cells, and protein expression of CTHRC1 was detected by Western blot after $48 \mathrm{~h}$ of transfection. ${ }^{* *, 8 \&} P<.01$.

CTHRC1 Was Targeted by miR-509-3p in Melanoma Cells Target genes of miR-509-3p associated with biological effects in melanoma were screened and identified. CTHRC1 contains potential binding sites for miR-509-3p (Figure 3A). The results demonstrated that luciferase activity of CTHRC1-WT was decreased upon miR-509-3p mimic treatment compared to that of NC mimic treatment in SK-MEL-28 $(P<.001)$ and WM115 $(P=.004)$ cells (Figure 3B), while CTHRC1 mutation abolished such a suppressive effect of miR-509-3p (Figure 3B), suggesting that miR-509-3p directly binds to CTHRC1. Moreover, miR-509-3p overexpression directly decreased the CTHRC1 level, whereas miR-509-3p inhibition increased the expression of SK-MEL-28 $(P<.001$ and $P<.001)$ and WM115 $(P<.001$ and $P<.001)$ cells (Figure 3C). Taken together, data obtained so far illustrated that CTHRC1 was targeted by miR-509-3p in melanoma cells.

\section{miR-509-3p Suppressed Malignant Metastasis of Melanoma Cells Through Regulation of CTHRC1}

To determine the effect of miR-509-3p/CTHRC1 axis in the metastasis of melanoma, pcDNA-CTHRC1 and miR-509-3p mimic were cotransfected into SK-MEL-28 and WM115 cells. Western blot analysis showed that CTHRC1 overexpression decreased protein levels of $\alpha$-catenin $(P<.001)$ and E-cadherin $(P<.001)$, whereas enhanced vimentin $(P<.001)$ and fibronectin $(P<.001)$ (Figure 4A). However, cotransfection using pcDNACTHRC1 and miR-509-3p mimic reversed CTHRC1-induced decrease of $\alpha$-catenin $(P<.001)$ and E-cadherin $(P<.001)$ levels, as well as restored the CTHRC1-mediated increase of vimentin $(P<.001)$ and fibronectin $(P<.001)$ (Figure 4A). Moreover, elevated CTHRC1 expression promoted migration (Figure 4B) and invasion (Figure 4B) of SK-MEL-28 and WM115 $(P<.001)$ cells. miR-509-3p could attenuate the promotive effects of CTHRC1 on cell migration (Figure 4B) and invasion (Figure 4C) in SK-MEL-28 and WM115 $(P<.001)$ cells. Collectively, miR-509-3p suppressed the malignant metastasis of melanoma cells through the regulation of CTHRC1.

\section{DISCUSSION}

Distant metastasis is a major obstacle for the effective treatment of melanoma, metastasis, and invasion, which are the most important reasons for the death of patients due to melanoma.$^{14}$ miRNAs, due to their ability to modulate melanoma cell growth, have been considered as new therapeutic strategies for melanoma. ${ }^{7}$ Although miR-509-3p was shown to be responsible for suppressing tumor cell metastasis, the exact role of miR-509-3p in melanoma progression remained to be investigated.

Melanoma is highly curable in the early stages; however, melanoma often develops metastasis devoid of effective diagnostic biomarkers. ${ }^{15}$ Therefore, it is important to discover diagnostic or prognostic biomarkers for melanoma. miRNAs have been reported to be related to the diagnosis and prognosis of melanoma, which could contribute to personalized treatment. ${ }^{16}$ Dysregulation of the miR-506-514 cluster, including miR-506-3p and miR-509-3p, 

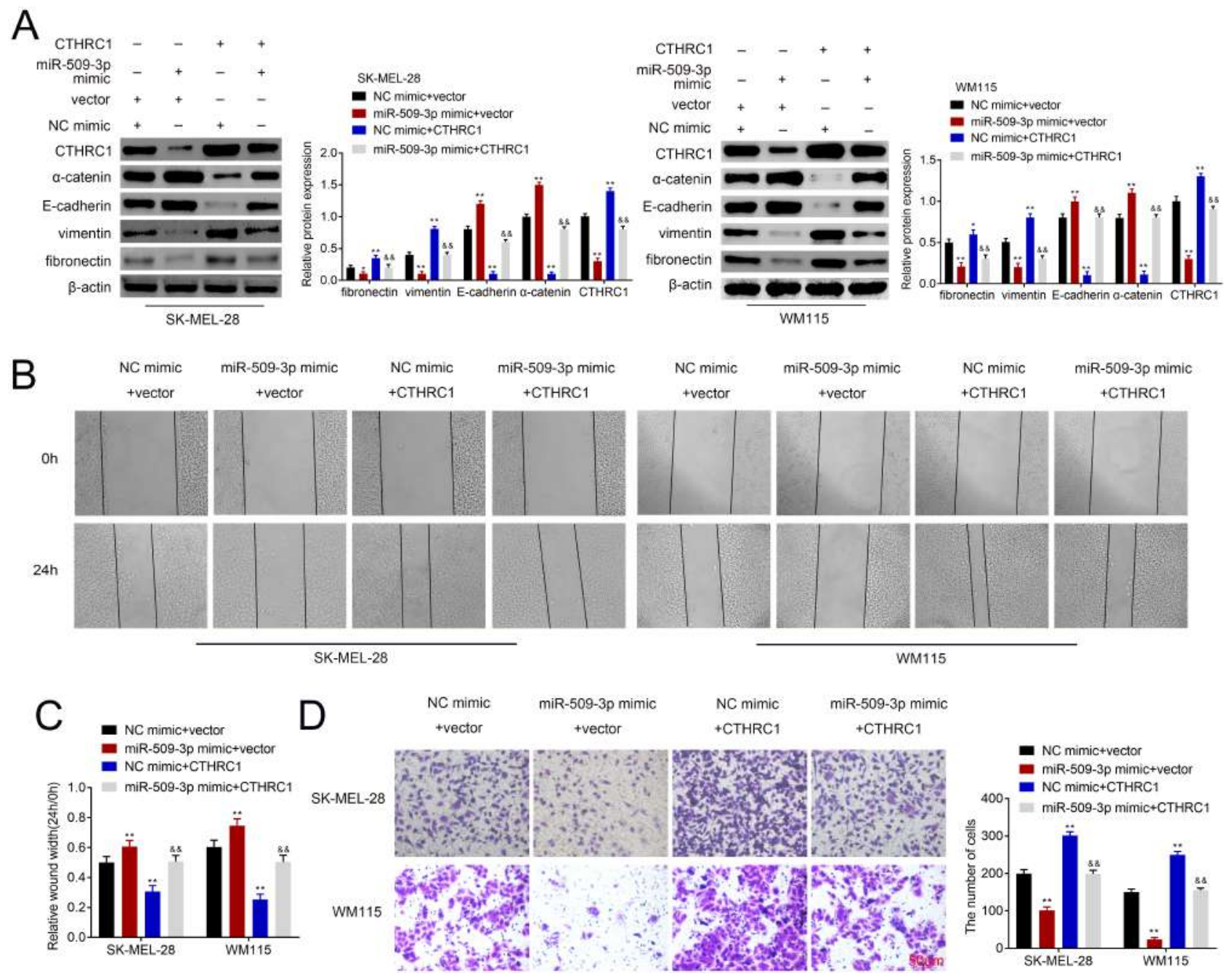

FIG. 4. (A-D). miR-509-3p suppressed malignant metastasis of melanoma cells through regulation of CTHRC1. (A) Effect of pcDNA-CTHRC1 and miR-509-3p mimic on protein expression of CTHRC1, $\alpha$-catenin, E-cadherin, vimentin, and fibronectin in SK-MEL-28 and WM115 cells were detected by Western blot after $48 \mathrm{~h}$ of transfection. (B) Effect of pcDNA-CTHRC1 and miR-509-3p mimic on cell migration in SK-MEL-28 and WM115 cells were detected by wound healing after $48 \mathrm{~h}$ of transfection. (C) Effect of pcDNA-CTHRC1 and miR-509-3p mimic on cell invasion in SK-MEL-28 and WM115 cells were detected by transwell assay after $48 \mathrm{~h}$ of transfection. ${ }^{* *, \& \&} P<.01$

was found in metastatic cutaneous melanoma. ${ }^{17}$ miR-509-3p was reduced in melanoma patients, and such different expression levels could discriminate between non-melanoma controls and melanoma patients at different stages. ${ }^{16}$ Moreover, glypican 6 was upregulated in melanoma cell lines and considered as a biomarker for cutaneous melanoma, whose expression was suppressed by miR-509-3p. ${ }^{18}$ We observed that miR-509-3p was dysregulated in melanoma cells, and in this study, the relationship between overall survival or clinico-pathological parameters of patients with melanoma and miR-509-3p expression levels should be further investigated, with the aim to provide diagnostic or prognostic roles of miR-509-3p in melanoma.

Functional assays revealed that miR-509-3p could suppress melanoma cell migration and invasion, suggesting the anticancer role in melanoma. Epithelial-mesenchymal transition in melanoma cells is often accompanied by the transformation of epithelial cells into invasive mesenchymal cells, and promotion of epithelial-mesenchymal transition could contribute to metastasis of melanoma. ${ }^{19}$ Suppression of epithelial-mesenchymal transition facilitated the repressive role of miR-204-3p on melanoma cells. ${ }^{20}$ This study indicated that miR-509-3p decreased the expressions of vimentin and fibronectin, whereas increased the expression of $\alpha$-catenin and E-cadherin in melanoma cells, thus suppressing metastasis of melanoma cells. Transcription factors, including TWIST1, Slug, and Snail, have been shown to promote epithelialmesenchymal transition in tumors. ${ }^{21}$ Since miR-509-3p could target epithelial-mesenchymal transition regulator, TWIST1, in ovarian cancer, ${ }^{9}$ whether TWIST1 was involved in miR-509-3p-mediated 
epithelial-mesenchymal transition in melanoma cells should be investigated in future studies.

CTHRC1 was found to be enhanced in metastatic melanoma specimens compared to nonmetastatic lesions ${ }^{12}$ and promoted melanoma cell survival. ${ }^{22}$ CTHRC1 knockdown retarded the progression of melanoma cells. ${ }^{23}$ We found that CTHRC1 overexpression contributed to the progression of melanoma cells. Previous research reported that the transforming growth factor could stimulate CTHRC1 to modulate Smad2/3, Wnt or Src, and Erk pathways, thus enhancing epithelial-mesenchymal transition to facilitate cancer metastasis. ${ }^{24}$ Elevated CTHRC1 expression in melanoma cells also promotes epithelial-mesenchymal transition with increased vimentin and fibronectin and decreased $\alpha$-catenin and E-cadherin. CTHRC1 was found to be involved in miR-134- ${ }^{25}$ or miR-155-26-mediated melanoma tumorigenesis. This study first confirmed that miR-509-3p could directly target CTHRC1 and negatively regulated its protein expression. Moreover, CTHRC1 overexpression counteracted the repressive effects of miR-509-3p on melanoma cell migration, invasion, and epithelial-mesenchymal transition. Furthermore, the detailed downstream signaling pathways involved in progression regulation by miR-509-3p/CTHRC1 axis need to be further investigated.

In conclusion, this study reported miR-509-3p was a tumor suppressor in melanoma cells, modulating tumor progression through inhibiting CTHRC1 expression. Taken together, our results provide insights into miR-509-3p as a promising therapeutic agent in melanoma progression from bench to clinic.

Ethics Committee Approval: N/A.

Patient Consent for Publication: N/A

Data-sharing Statement: Data sharing is not applicable to this article as no new data were created or analyzed in this study.

Author Contributions: Concept - Y.J.; Design - Y.J.; Supervision - K.Y.; Resources K.Y.; Materials - Y.S.; Data Collection and/or Processing - Y.S.; Writing - K.Y., Y.S., Y.J.

Conflict of Interest: The authors have no conflicts of interest to declare.

Funding: The authors declared that this study had received no financial support.

\section{REFERENCES}

1. Jang YC, Liang SJ. Vaginal primary malignant melanoma of a Chinese woman: an exceptionally rare yet lethal case report and literature review. Eur J Gynaecol Oncol. 2018;39:327-330

2. Fraihat A, Alatrash L, Abbasi R, et al. Inhibitory effects of methanol extracts of selected plants on the proliferation of two human melanoma cell lines. Trop J Pharm Res. 2018;17(6):1081-1086. [CrossRef]

3. Segura MF, Belitskaya-Lévy I, Rose AE, et al. Melanoma microRNA signature predicts post-recurrence survival. Clin Cancer Res. 2010;16(5):1577-1586. [CrossRef]

4. Shukla GC, Singh J, Barik S. MicroRNAs: processing, maturation, target recognition and regulatory functions. Mol Cell Pharmacol. 2011;3(3):83-92.
5. Lee YS, Dutta A. MicroRNAs in cancer. Annu Rev Pathol, 2009:4:199-227. [CrossRef]

6. Mione M, Bosserhoff A. MicroRNAs in melanocyte and melanoma biology. Pigment Cell Melanoma Res. 2015;28(3):340-354. [CrossRef]

7. Lorusso C, De Summa S, Pinto R, Danza K, Tommasi S. miRNAs as key players in the management of cutaneous melanoma. Cells. 2020;9(2):415. [CrossRef]

8. Zhai Q, Zhou L, Zhao C, et al. Identification of miR-508-3p and miR-509-3p that are associated with cell invasion and migration and involved in the apoptosis of renal cell carcinoma. Biochem Biophys Res Commun. 2012;419(4):621-626. [CrossRef]

9. Pan Y, Robertson G, Pedersen L, et al. miR-509-3p is clinically significant and strongly attenuates cellular migration and multi-cellular spheroids in ovarian cancer. Oncotarget. 2016;7(18):25930-25948. [CrossRef]

10. Díaz-Martínez M, Benito-Jardón L, Alonso L, et al. miR-204-5p and miR-211-5p contribute to BRAF inhibitor resistance in melanoma. Cancer Res. 2018;78(4):10171030. [CrossRef]

11. Stark MS, Klein K, Weide B, et al. The prognostic and predictive value of melanomarelated microRNAs using tissue and serum: a microRNA expression analysis. EBiomedicine. 2015;2(7):671-680. [CrossRef]

12. Tang L, Dai DL, Su M, et al. Aberrant expression of collagen triple helix repeat containing 1 in human solid cancers. Clin Cancer Res. 2006;12(12):3716-3722. [CrossRef]

13. Jiang N, Cui Y, Liu J, et al. Multidimensional roles of collagen triple helix repeat containing 1 (CTHRC1) in malignant cancers. J Cancer. 2016;7(15):2213-2220. [CrossRef]

14. Luo Z, Zhang X, Zeng W, et al. TRAF6 regulates melanoma invasion and metastasis through ubiquitination of basigin. Oncotarget. 2016;7(6):7179-7192. [CrossRef]

15. Scott KL, Nogueira C, Heffernan TP, et al. Proinvasion metastasis drivers in earlystage melanoma are oncogenes. Cancer Cell. 2011;20(1):92-103. [CrossRef]

16. Mumford SL, Towler BP, Pashler AL, et al. Circulating microRNA biomarkers in melanoma: tools and challenges in personalised medicine. Biomolecules. 2018;8(2):21. [CrossRef]

17. Philippidou D, Schmitt M, Moser D, et al. Signatures of microRNAs and selected microRNA target genes in human melanoma. Cancer Res. 2010;70(10):4163-4173. [CrossRef]

18. Li Y, Li M, Shats I, et al. Glypican 6 is a putative biomarker for metastatic progression of cutaneous melanoma. PLoS ONE. 2019;14(6):e0218067. [CrossRef]

19. Hodorogea A, Calinescu A, Antohe M, et al. Epithelial-mesenchymal transition in skin cancers: a review. Anal Cell Pathol. 2019;2019:3851576. [CrossRef]

20. Song J, Chen X, Zhang L, Song D, Xiong H. MicroRNA-204-3p modulates epithelialmesenchymal transition by targeting paired box gene 2 in human melanoma A-375 cells. Transl Cancer Res. 2019;8(5):2032-2043. [CrossRef]

21. Zhang J, Ma L. MicroRNA control of epithelial-mesenchymal transition and metastasis. Cancer Metastasis Rev. 2012;31(3-4):653-662. [CrossRef]

22. Park EH, Kim S, Jo JY, et al. Collagen triple helix repeat containing-1 promotes pancreatic cancer progression by regulating migration and adhesion of tumor cells. Carcinogenesis. 2013;34(3):694-702. [CrossRef]

23. Eriksson J, Le Joncour V, Nummela P, et al. Gene expression analyses of primary melanomas reveal CTHRC1 as an important player in melanoma progression. Oncotarget. 2016;7(12):15065-15092. [CrossRef]

24. Turkar S, Ramaswamy A, Ostwal V. A step ahead on CTHRC1, and not just reinventing the wheel! Chin Clin Oncol. 2019;8(S1):S17. [CrossRef]

25. Li Y, Fu Y, Gao Y, et al. microRNA-134 inhibits melanoma growth and metastasis by negatively regulating collagen triple helix repeat containing-1 (CTHRC1). Int $J$ Clin Exp Pathol. 2018;11(9):4319-4330.

26. Li Y, Zhang $\mathrm{Y}$, Ma C, et al. Overexpression of CTHRC1 in human melanoma promotes tumorigenesis targeted by miRNA155. Int $J$ Clin Exp Pathol. 2017;10(8):8199-8210. 